\title{
CURVATURE PROPERTIES OF TWISTOR SPACES
}

\author{
JOHANN DAVIDOV AND OLEG MUSHKAROV \\ Dedicated to Professor Armen Sergeev on the occasion of his 70th birthday
}

\begin{abstract}
In this paper we review some results on the Riemannian and almost Hermitian geometry of twistor spaces of oriented Riemannian 4-manifolds with emphasis on their curvature properties.

2010 Mathematics Subject Classification. Primary 53C28, Secondary 53C56.

Key words: twistor spaces, almost Hermitian structures, Riemannian and Hermitian curvatures of twistor spaces.
\end{abstract}

\section{INTRODUCTION}

The twistor theory has its origin in Mathematical Physics. Inspired by the Penrose program [81, 82, Atiyah, Hitchin and Singer [16] developed this theory on oriented Riemannian 4-manifolds. They defined the twistor space of such a manifold $M$ as the 2 -sphere bundle $\mathcal{Z}$ on $M$ whose fiber at any point $p \in M$ consists of all complex structures on the tangent space $T_{p} M$ compatible with the metric and the opposite orientation of $M$. The 6 -manifold $\mathcal{Z}$ admits two natural almost complex structures $\mathcal{J}_{1}$ and $\mathcal{J}_{2}$ introduced, respectively, by Atiyah, Hitchin, and Singer [16] and by Eells and Salamon [47. The almost complex structure $\mathcal{J}_{2}$ is never integrable but it plays an important role in the theory of harmonic maps. The almost complex structure $\mathcal{J}_{1}$ is integrable if and only if the base manifold $M$ is self-dual, i.e. its Weyl conformal tensor $\mathcal{W}$ satisfies the equation $* \mathcal{W}=\mathcal{W}$, where $*$ is the Hodge star-operator [19. So, in the case when $M$ is self-dual, $\mathcal{Z}$ is a complex 3-manifold and each fibre $\mathcal{Z}_{p}=\pi^{-1}(p), p \in M$, is a complex submanifold of $\mathcal{Z}$ biholomorphic to $\mathbb{C P}^{1}$. The normal bundle of $\mathcal{Z}_{p}$ is biholomorphically equivalent to $H \oplus H$, where $H$ is the positive Hopf bundle on $\mathbb{C P}^{1}$. The antipodal map $j \rightarrow-j$ on each fibre induces an anti-biholomorphic involution of $\mathcal{Z}$ without fixed points.

The above construction depends only on the conformal class of the given metric on $M$ and, conversely, the complex structure of the twistor space determines the self-dual conformal structure on $M$. More precisely, let $\mathcal{Z}$ be a complex 3-manifold with the following properties: (1) $\mathcal{Z}$ is fibered by projective lines whose normal bundle is isomorphic to $H \oplus H$; (2) $\mathcal{Z}$ possesses a free anti-holomorphic involution which transforms each fibre to itself. Then $\mathcal{Z}$ is the twistor space of a self-dual manifold $M$ ([16, see also [19]). The described correspondence between self-dual manifolds and twistor spaces is called the Penrose correspondence and it has been used for years to study the conformal geometry of four-manifolds by means of complex geometry techniques.

The authors are partially supported by the National Science Fund, Ministry of Education and Science of Bulgaria under contract DN 12/2 . 
The twistor space $\mathcal{Z}$ admits a 1-parameter family of Riemannian metrics which are compatible with the almost complex structures $\mathcal{J}_{1}$ and $\mathcal{J}_{2}$ and the natural projection $\pi: \mathcal{Z} \rightarrow M$ is a Riemannian submersion [19]. These natural almost Hermitian structures are very interesting geometric objects in their own right whose differential geometric properties have been studied by many authors, to cite here just a few [14, 42, 48, 49, 50, 51, 62, 63, 83.

Motivated by open questions in non-Kähler geometry in a series of papers 77 , 36. 35, 33, 34, 39, 37, 9, 2], the authors studied the twistor spaces of oriented Riemannian 4-manifolds as a source of examples of almost Hermitian 6-manifolds with interesting geometric properties. In the present paper we review some of these results with emphasis on the curvature properties of twistor spaces.

\section{Table of contents}

2. Preliminaries

3. Riemannian curvature of twistor spaces

3.1. Riemannian sectional curvature

3.2. Ricci curvature

4. Twistor spaces with Hermitian Ricci tensor

5. Kähler curvature identities on twistor spaces

6. *-Einstein twistor spaces

7. Curvature properties of the Chern connection on twistor spaces

8. Holomorphic curvatures of twistor spaces

8.1. Holomorphic bisectional curvature

8.2. Orthogonal bisectional curvatures

8.3. Hermitian bisectional curvature

Acknowledgements. The authors would like to thank the editors of this volume for the invitation to submit a paper in honour of Professor Armen Sergeev, our dear friend and colleague.

\section{Preliminaries}

Let $(M, g)$ be an oriented (connected) Riemannian manifold of dimension four. The metric $g$ induces a metric on the bundle of two-vectors $\pi: \Lambda^{2} T M \rightarrow M$ by the formula

$$
g\left(v_{1} \wedge v_{2}, v_{3} \wedge v_{4}\right)=\frac{1}{2} \operatorname{det}\left[g\left(v_{i}, v_{j}\right)\right],
$$

the factor $1 / 2$ being chosen in accordance with [36. Let $*: \Lambda^{k} T M \rightarrow \Lambda^{4-k} M$, $k=0, \ldots, 4$, be the Hodge star operator. For $k=2$, it is an involution of $\Lambda^{2} T M$, thus we have the orthogonal decomposition

$$
\Lambda^{2} T M=\Lambda_{-}^{2} T M \oplus \Lambda_{+}^{2} T M
$$

where $\Lambda_{ \pm}^{2} T M$ are the subbundles of $\Lambda^{2} T M$ corresponding to the eigenvalues \pm 1 of the operator $*$. Given a local oriented orthonormal frame $\left(E_{1}, E_{2}, E_{3}, E_{4}\right)$ of $T M$ we set

(1) $s_{1}^{ \pm}=E_{1} \wedge E_{2} \pm E_{3} \wedge E_{4}, \quad s_{2}^{ \pm}=E_{1} \wedge E_{3} \pm E_{4} \wedge E_{2}, \quad s_{3}^{ \pm}=E_{1} \wedge E_{4} \pm E_{2} \wedge E_{3}$.

Then $\left(s_{1}^{ \pm}, s_{2}^{ \pm}, s_{3}^{ \pm}\right)$is a local orthonormal frame of $\Lambda_{ \pm}^{2} T M$. This frame defines an orientation on $\Lambda_{ \pm}^{2} T M$ which does not depend on the choice of the frame $\left(E_{1}, E_{2}, E_{3}, E_{4}\right)$ (see, for example, 32]). We call this orientation "canonical".

For every $a \in \Lambda^{2} T M$, define a skew-symmetric endomorphism of $T_{\pi(a)} M$ by 


$$
g\left(K_{a} X, Y\right)=2 g(a, X \wedge Y), \quad X, Y \in T_{\pi(a)} M .
$$

Denoting by $G$ the standard metric $-\frac{1}{2} \operatorname{Trace}(P Q)$ on the space of skew-symmetric endomorphisms, we have $G\left(K_{a}, K_{b}\right)=2 g(a, b)$ for $a, b \in \Lambda^{2} T M$. The assignment $a \rightarrow K_{a}$ is the standard isomorphism of the bundle $\Lambda^{2} T M$ with the bundle $A(T M)$ of $g$-skew-symmetric endomorphism of $T M$. Let $\times$ be the usual vector cross product on the oriented 3-dimensional vector space $\Lambda_{ \pm}^{2} T_{p} M, p \in M$, endowed with the metric $g$. If $a, b \in \Lambda_{ \pm}^{2} T_{p} M$, the isomorphism $\Lambda^{2} T M \cong A(T M)$ sends $a \times b$ to $\pm \frac{1}{2}\left[K_{a}, K_{b}\right]$. In the case when $a \in \Lambda_{+}^{2} T_{p} M, b \in \Lambda_{-}^{2} T_{p} M$, the endomorphisms $K_{a}$ and $K_{b}$ of $T_{p} M$ commute. For $a, b \in \Lambda_{ \pm} T_{p} M$, we have

$$
K_{a} \circ K_{b}=-g(a, b) I d \pm K_{a \times b} .
$$

In particular, $K_{a}$ and $K_{b}, a, b \in \Lambda_{ \pm} T_{p} M$, anti-commute if and only if $a$ and $b$ are orthogonal.

If $\sigma \in \Lambda^{2} T M$ is of unit length, then $K_{\sigma}$ is a complex structure on the vector space $T_{\pi(\sigma)} M$ compatible with the metric $g$, i.e., $g$-orthogonal. Conversely, the 2-vector $\sigma$ dual to one half of the Kähler 2-form of such a complex structure is a unit vector in $\Lambda^{2} T M$. Therefore the unit sphere bundle $\mathcal{Z}$ of $\Lambda^{2} T M$ parametrizes the complex structures on the tangent spaces of $M$ compatible with the metric $g$. This bundle is called the twistor space of the Riemannian manifold $(M, g)$. Since $M$ is oriented, the manifold $\mathcal{Z}$ has two connected components $\mathcal{Z}_{ \pm}$called the positive and negative twistor spaces of $(M, g)$. These are the unit sphere subbundles of $\Lambda_{ \pm}^{2} T M$. The bundle $\pi: \mathcal{Z}_{ \pm} \rightarrow M$ parametrizes the complex structures on the tangent spaces of $M$ compatible with the metric and the \pm orientation via the correspondence $\mathcal{Z}_{ \pm} \ni \sigma \rightarrow K_{\sigma}$.

The connection $\nabla$ on $\Lambda^{2} T M$ induced by the Levi-Civita connection of $M$ preserves the bundles $\Lambda_{ \pm}^{2} T M$, so it induces a metric connection on each of them denoted again by $\nabla$. The horizontal distribution of $\Lambda_{ \pm}^{2} T M$ with respect to $\nabla$ is tangent to the twistor space $\mathcal{Z}_{ \pm}$. Thus, we have the decomposition $T \mathcal{Z}_{ \pm}=\mathcal{H} \oplus \mathcal{V}$ of the tangent bundle of $\mathcal{Z}_{ \pm}$into horizontal and vertical components. The vertical space $\mathcal{V}_{\sigma}=\left\{V \in T_{\sigma} \mathcal{Z}_{ \pm}: \pi_{*} V=0\right\}$ at a point $\sigma \in \mathcal{Z}_{ \pm}$is the tangent space to the fibre of $\mathcal{Z}_{ \pm}$through $\sigma$. If we consider $T_{\sigma} \mathcal{Z}_{ \pm}$as a subspace of $T_{\sigma}\left(\Lambda_{ \pm}^{2} T M\right)$, then the space $\mathcal{V}_{\sigma}$ is the orthogonal complement of $\sigma$ in $\Lambda_{ \pm}^{2} T_{\pi(\sigma)} M$.

The 6-manifold $\mathcal{Z}_{ \pm}$admits two almost complex structures $\mathcal{J}_{1}$ and $\mathcal{J}_{2}$ introduced, respectively, by Atiyah, Hitchin, and Singer [16] and by Eells and Salamon [47. For $\sigma \in \mathcal{Z}_{ \pm}$, the horizontal space $\mathcal{H}_{\sigma}$ is isomorphic to the tangent space $T_{\pi(\sigma)} M$ via the differential $\pi_{* \sigma}$, and the structures $\mathcal{J}_{1}$ and $\mathcal{J}_{2}$ on the space $\mathcal{H}_{\sigma}$ are both defined as the lift to $\mathcal{H}_{\sigma}$ of the complex structure $K_{\sigma}$ on $T_{\pi(\sigma)} M$. The vertical space $\mathcal{V}_{\sigma}$ is tangent to the unit sphere in the 3 -dimensional vector space $\left(\Lambda_{ \pm}^{2} T_{\pi(\sigma)} M, g\right)$, and we denote by $\mathcal{J}_{\sigma}$ the standard complex structure of the unit sphere restricted to $\mathcal{V}_{\sigma}$. It is given by

$$
\mathcal{J}_{\sigma} V= \pm(\sigma \times V), \quad V \in \mathcal{V}_{\sigma}
$$

On a vertical space $\mathcal{V}_{\sigma}, \mathcal{J}_{1}$ is defined to be the complex structure $\mathcal{J}_{\sigma}$ of the fibre through $\sigma$, while $\mathcal{J}_{2}$ is defined as the conjugate complex structure, i.e., $\mathcal{J}_{2} \mid \mathcal{V}_{\sigma}=$ 
$-\mathcal{J}_{\sigma}$. Thus, for $\sigma \in \mathcal{Z}_{ \pm}$,

$$
\begin{gathered}
\mathcal{J}_{n}\left|\mathcal{H}_{\sigma}=\left(\pi_{*} \mid \mathcal{H}_{\sigma}\right)^{-1} \circ K_{\sigma} \circ \pi_{*}\right| \mathcal{H}_{\sigma} \\
\mathcal{J}_{n} V= \pm(-1)^{n+1}(\sigma \times V) \text { for } V \in \mathcal{V}_{\sigma}, \quad n=1,2 .
\end{gathered}
$$

It is a result of Eells and Salamon [4] that the almost complex structure $\mathcal{J}_{2}$ is never integrable, so it does not come from a complex structure. Nevertheless, $\mathcal{J}_{2}$ is very useful for constructing harmonic maps. The integrability condition for $\mathcal{J}_{1}$ has been found by Atiyah, Hitchin, and Singer [16. To state their result, we first recall the well-known curvature decomposition in dimension four. Note that for the curvature tensor $R$, we adopt the following definition: $R(X, Y)=$ $\nabla_{[X, Y]}-\left[\nabla_{X}, \nabla_{Y}\right]$. The curvature operator $\mathcal{R}$ corresponding to the curvature tensor is the endomorphism of $\Lambda^{2} T M$ defined by

$$
g(\mathcal{R}(X \wedge Y), Z \wedge U)=g(R(X, Y) Z, U), \quad X, Y, Z, U \in T M .
$$

Denote by $\rho$ the Ricci tensor of $(M, g)$ and by $A: T M \rightarrow T M$ its Ricci operator, $g(A(X), Y)=\rho(X, Y)$. Then the endomorphism $\mathcal{B}: \Lambda^{2} T M \rightarrow \Lambda^{2} T M$ corresponding to the traceless Ricci tensor is given by

$$
\mathcal{B}(X \wedge Y)=A(X) \wedge Y+X \wedge A(Y)-\frac{s}{2} X \wedge Y,
$$

where $s$ is the scalar curvature. Note that $\mathcal{B}$ sends $\Lambda_{ \pm}^{2} T M$ into $\Lambda_{\mp}^{2} T M$. Let $\mathcal{W}: \Lambda^{2} T M \rightarrow \Lambda^{2} T M$ be the endomorphism corresponding to the Weyl conformal tensor. Denote the restriction of $\mathcal{W}$ to $\Lambda_{ \pm}^{2} T M$ by $\mathcal{W}_{ \pm}$, so $\mathcal{W}_{ \pm}$sends $\Lambda_{ \pm}^{2} T M$ to $\Lambda_{ \pm}^{2} T M$ and vanishes on $\Lambda_{\mp}^{2} T M$.

It is well known that the curvature operator decomposes as (87, 19, Chapter $1 \mathrm{H}])$

$$
\mathcal{R}=\frac{s}{6} I d+\mathcal{B}+\mathcal{W}_{+}+\mathcal{W}_{-}
$$

Note that this differs from [19] by a factor of $1 / 2$ because of the factor $1 / 2$ in our definition of the induced metric on $\Lambda^{2} T M$. The Riemannian manifold $(M, g)$ is Einstein exactly when $\mathcal{B}=0$. It is called self-dual (anti-self-dual) if $\mathcal{W}_{-}=0\left(\mathcal{W}_{+}=0\right)$. The self-duality (anti-self-duality) condition is invariant under conformal changes of the metric since the Weyl tensor is so. Note also that changing the orientation of $M$ interchanges the roles of $\Lambda_{-}^{2} T M$ and $\Lambda_{+}^{2} T M$ (respectively, of $\mathcal{Z}_{-}$and $\mathcal{Z}_{+}$), hence the roles of $\mathcal{W}_{-}$and $\mathcal{W}_{+}$.

The famous Atiyah-Hitchin-Singer theorem [16] states that the almost complex structure $\mathcal{J}_{1}$ on $\mathcal{Z}_{-}$(resp. $\mathcal{Z}_{+}$) is integrable if and only if $(M, g)$ is self-dual (resp. anti-self-dual).

The twistor space $\mathcal{Z}_{ \pm}$of an oriented Riemannian 4-manifold $(M, g)$ admits a natural 1-parameter family of Riemannian metrics $h_{t}$ defined by

$$
h_{t}=\pi^{*} g+t g^{v}
$$

where $t>0$ and $g^{v}$ is the restriction of the metric of $\Lambda^{2} T M$ on the vertical distribution $\mathcal{V}$. Then $\pi:\left(\mathcal{Z}_{ \pm}, h_{t}\right) \rightarrow(M, g)$ is a Riemannian submersion with totally geodesic fibres, and the almost-complex structures $\mathcal{J}_{1}$ and $\mathcal{J}_{2}$ are compatible with the metrics $h_{t}$. The Gray-Hervella classes [59] of the almost Hermitian structures $\left(h_{t}, \mathcal{J}_{n}\right), t>0, n=1,2$, have been determined in [77. 


\section{Riemannian CURVATURE OF TWISTOR SPACES}

The O'Neill formulas [79], 19, Ch.9 G] can be used to obtain coordinate-free formulas for various curvatures of the metric $h_{t}$ on the twistor space in terms of the curvature of its base manifold $M$. This is done in [36] in the case when $\operatorname{dim} M=4$ and in 31] for the general twistor space of partially complex structures ( $f$-structures) on a Riemannian manifold of any dimension $\geq 3$. We shall discuss here the most interesting case of the negative twistor space of an oriented four dimensional Riemannian manifold. The reason to choose the negative twistor space is connected with the Atiyah-Hitchin-Singer theorem mentioned above. As smooth manifolds, the positive and the negative twistor spaces of $\mathbb{C P}^{2}$ coincide with the complex flag manifold $F_{1,2}$. The Atiyah-Hitchin-Singer almost complex structure on the negative twistor space of $\mathbb{C P}^{2}$ is integrable and coincides with the standard complex structure of $F_{1,2}$, while it is not integrable on the positive twistor space.

In what follows, $(M, g)$ will denote an oriented Riemannian manifold of dimension four, and $\mathcal{Z}$ will stand for its negative twistor space $\mathcal{Z}_{-}$.

3.1. Riemannian sectional curvature. Let $(M, g)$ be an oriented Riemannian 4-manifold with Levi-Civita connection $\nabla$ and Riemannian curvature tensor $R$. For any $t>0$ denote by $R_{t}$ the Riemannian curvature tensor of the metric $h_{t}$ on the twistor space $\mathcal{Z}$ of $(M, g)$. Applying the O'Neill formulas [79] for the Riemannian submersion $\pi:\left(\mathcal{Z}, h_{t}\right) \rightarrow(M, g)$, one can compute the Riemannina sectional curvature of $\left(\mathcal{Z}, h_{t}\right)$.

Proposition 1. ([36]) Let $E, F \in T_{\sigma} \mathcal{Z}, X=\pi_{*} E, Y=\pi_{*} F, V=\mathcal{V} E$ and $W=\mathcal{V} F$. Then

$$
\begin{gathered}
h_{t}\left(R_{t}(E \wedge F) E, F\right)=g(R(X \wedge Y) X, Y)-\operatorname{tg}\left(\left(\nabla_{X} \mathcal{R}\right)(X \wedge Y), \sigma \times W\right) \\
+\operatorname{tg}\left(\left(\nabla_{Y} \mathcal{R}\right)(X \wedge Y), \sigma \times V\right)-3 \operatorname{tg}(\mathcal{R}(\sigma), X \wedge Y) g(\sigma \times V, W) \\
-t^{2} g(R(\sigma \times V) X, R(\sigma \times W) Y)+\frac{t^{2}}{4}\|R(\sigma \times W) X+R(\sigma \times V) Y\|^{2} \\
-\frac{3 t}{4}\|R(X \wedge Y) \sigma\|^{2}+t\left(\|V\|^{2}\|W\|^{2}-g(V, W)^{2}\right) .
\end{gathered}
$$

In the case when the base manifold $(M, g)$ is self-dual and Einstein the above formula takes an apparently simple form.

Corollary 1. Let $(M, g)$ be a self-dual Einstein manifold with scalar curvature s. Then

$$
\begin{gathered}
h_{t}\left(R_{t}(E \wedge F) E, F\right)=g(R(X \wedge Y) X, Y)-\frac{t s}{2} g(\sigma, X \wedge Y) g(\sigma \times V, W) \\
-\frac{1}{2}\left(\frac{t s}{12}\right)^{2} g(X, Y) g(V, W)+3\left(\frac{t s}{12}\right)^{2} g(X \wedge Y, V \wedge W) \\
+\left(\frac{t s}{24}\right)^{2}\left(\|X\|^{2}\|W\|^{2}+\|Y\|^{2}\|V\|^{2}\right) \\
-6 t\left(\frac{s}{24}\right)^{2}\left(\|X \wedge Y\|^{2}-2 g(\sigma, X \wedge Y)^{2}\right) \\
+t\left(\|V\|^{2}\|W\|^{2}-g(V, W)^{2}\right) .
\end{gathered}
$$


3.2. Ricci curvature. The study of the Ricci curvature of a twistor space is based on the following explicit formula for the Ricci tensor which is a consequence of Proposition 1.

Proposition 2. (36]) Let $\rho_{t}$ be the Ricci tensor of the twistor space $\left(\mathcal{Z}, h_{t}\right)$. If $E \in T_{\sigma} \mathcal{Z}, X=\pi_{*} E$, and $V=\mathcal{V} E$, then

$$
\begin{aligned}
\rho_{t}(E, E) & =\rho(X, X)+\operatorname{tg}(\delta \mathcal{R}(X), \sigma \times V)+\frac{t^{2}}{4}\|\mathcal{R}(\sigma \times V)\|^{2} \\
& +\frac{t}{2}\left\|\imath_{X} \circ \mathcal{R}(\sigma)\right\|^{2}-\frac{t}{2}\left\|\imath_{X} \circ \mathcal{R}_{-}\right\|^{2}+\|V\|^{2},
\end{aligned}
$$

where $\rho$ is the Ricci tensor of $(M, g), \delta \mathcal{R}$ is the co-differential of $\mathcal{R}, \mathcal{R}_{-}$is the restriction of $\mathcal{R}$ on $\Lambda_{-}^{2} T M$, and $\imath_{X}: \Lambda^{2} T M \rightarrow T M$ is the interior product.

Taking the trace of $\rho_{t}$, we obtain the following formula for the scalar curvature $s_{t}$ of the twistor space $\left(\mathcal{Z}, h_{t}\right)$.

Corollary 2. ([36]) Let $s$ be the scalar curvature of $(M, g)$. Then

$$
s_{t}(\sigma)=s(\pi(\sigma))+\frac{t}{4}\left(\|\mathcal{R}(\sigma)\|^{2}-\left\|\mathcal{R}_{-}\right\|_{\pi(\sigma)}^{2}\right)+\frac{2}{t} .
$$

In the case when the base manifold of a twistor space is Einstein and self-dual, these formulas can significantly be simplified as follows.

Corollary 3. ([36]) If $(M, g)$ is Einstein and self-dual, the Ricci tensor $\rho_{t}$ of $\left(\mathcal{Z}, h_{t}\right)$ and its scalar curvature $s_{t}$ are given by

$$
\begin{gathered}
\rho_{t}(E, E)=\left[\frac{s}{4}-t\left(\frac{s}{12}\right)^{2}\right]\|X\|^{2}+\left[1+\left(\frac{t s}{12}\right)^{2}\right]\|V\|^{2}, \quad E=X_{\sigma}^{h}+V, \\
s_{t}=\frac{2}{t}+s-\frac{t}{72} s^{2} .
\end{gathered}
$$

As an application of Proposition 2, one can prove the following result of $\mathrm{T}$. Friedrich and R. Grunewald [49] about the Einstein condition on $\left(\mathcal{Z}, h_{t}\right)$.

Theorem 1. ([49, 36]) The Riemannian manifold $\left(\mathcal{Z}, h_{t}\right)$ is Einstein if and only if $(M, g)$ is a self-dual Einstein manifold with scalar curvature $s=6 / t$ or $s=12 / t$.

The next property of the Ricci tensor $\rho_{t}$ is an easy consequence of Corollary 3 .

Proposition 3. ([38]) If $(M, g)$ is Einstein and self-dual, the covariant derivative of the Ricci tensor $\rho_{t}$ of $\left(\mathcal{Z}, h_{t}\right)$ satisfies the identity

$$
\left(D_{E} \rho_{t}\right)(E, E)=0, \quad E \in T \mathcal{Z}
$$

where $D$ is the Levi-Civita connection of $h_{t}$. Moreover, $\rho_{t}$ is parallel if and only if $s t=6, s t=12$, or $s=0$.

Remark. Condition (6) for the Ricci tensor $\rho$ of a Riemannian manifold $(N, h)$ is known as the third Ledger condition [72, [98, Sec. 6.8]. It is easy to see by polarization that (6) is equivalent to the identity

$$
\left(\nabla_{X} \rho\right)(Y, Z)+\left(\nabla_{Y} \rho\right)(Z, X)+\left(\nabla_{Z} \rho\right)(X, Y)=0, \quad X, Y, Z \in T N
$$

where $\nabla$ is the Levi-Chivita connection of $(N, h)$. If this condition is satisfied, the manifold is real-analytic [90, and the scalar curvature is constant [30, Proposition 2.3]. Condition (6) appears in the study of the so-called D'Atri spaces which are characterized by the property that the geodesic symmetries preserve the volume 
up to sign [30]. It is one of the Einstein-like conditions introduced and studied by A. Gray [57, and discussed also in Besse's book [19, Sec. 16G] as an interesting generalization of the Einstein condition. We refer the reader to [19, 31, 57, 65, 67, 80, for examples of Riemannian manifolds satisfying condition (6). Proposition 3 , which gives twistorial examples of such manifolds, seems to be interesting in the case of negative scalar curvature of $(M, g)$ since the complete classification of compact Einstein self-dual manifolds with negative scalar curvature is not available yet. It has been conjectured by A. Vitter [97] that every such a manifold is a quotient of the unit ball in $\mathbb{C}^{2}$ with the metric of negative constant sectional curvature or the Bergman metric.

Corollary 3 can be used to show that an isometry of the twistor space preserves vertical, and hence horizontal, spaces. This implies the following.

Lemma 1. (31]) If $(M, g)$ is an Einstein and self-dual manifold with scalar curvature $s$, then every (local) isometry of the twistor space $\pi:\left(\mathcal{Z}, h_{t}\right) \rightarrow(M, g)$ descends to a (local) isometry of the metric $g$ provided $t s \neq 6$ and $t s \neq 12$.

Remarks. (31]) Suppose that the manifold $(M, g)$ is Einstein and self-dual, and $t s=6$ or $t s=12$.

1. Lemma 1 does not hold as there may exist an isometry of the twistor space of $(M, g)$ which does not descend to an isometry of $g$. For example, it is well-known that the twistor space $\mathcal{Z}$ of the sphere $S^{4}$ considered with its standard metric is the complex projective space $\mathbb{C P}^{3}$. To describe the twistor projection $\pi: \mathbb{C P}^{3} \rightarrow S^{4}$, it is convenient to identify $S^{4}$ with the quaternionic projective space $\mathbb{H}^{1}$. Then $\pi$ is given in homogeneous coordinates by $\left[z_{1}, z_{2}, z_{3}, z_{4}\right] \rightarrow\left[z_{1}+z_{2} \mathbf{j}, z_{3}+z_{4} \mathbf{j}\right]$. If $t s=12$, the metric $h_{t}$ is a multiple of the Fubini-Study metric. The map $\Psi: \mathbb{C P}^{3} \rightarrow \mathbb{C P}^{3}$ defined by $\Psi\left(\left[z_{1}, z_{2}, z_{3}, z_{4}\right]\right)=\left[\frac{1}{\sqrt{2}}\left(z_{1}+z_{2}\right), \frac{1}{\sqrt{2}}\left(z_{1}-z_{2}\right), z_{3}, z_{4}\right]$ is an isometry of the Fubini-Study metric which does not preserve all fibres of the twistor projection $\pi$.

2. The scalar curvature $s$ of $M$ is positive and, by a result of Hitchin 62 and of Friedrich and Kurke [50, see also [19, Theorem 13.30], $(M, g)$ is isometric to the sphere $S^{4}$ or the complex projective space $\mathbb{C P}^{2}$ with their standard metrics. In particular, the metric $g$ is homogeneous, hence all of the metrics $h_{t}$ on the twistor space are also homogeneous.

The latter remark and Lemma 1 give the following result which seems to be "folklore".

Proposition 4. (31]) Let $(M, g)$ be a complete Einstein self-dual manifold. The metric $h_{t}$ (with arbitrary $t$ ) on the twistor space $\mathcal{Z}$ is (locally) homogeneous if and only if the metric $g$ on the base manifold $M$ is (locally) homogeneous.

Proposition 3 and Lemma 1 imply the following

Proposition 5. ([31]) Let $M$ be an inhomogeneous Einstein self-dual 4-manifold with non-zero scalar curvature $s$. Then, for any $t>0$ with $t s \neq 6$ and $t s \neq 12$, the twistor space $\left(\mathcal{Z}, h_{t}\right)$ is non-homogeneous, has non-parallel Ricci tensor satisfying the third Ledger condition (6) and is not locally isometric to a Riemannian product.

Moreover, if $M$ is locally non-homogeneous, then so is its twistor space.

Remark. (31]) If the base manifold $M$ is locally homogeneous, so is its twistor space. There are a lot of examples of (non-compact) locally non-homogeneous, selfdual, Einstein manifolds with non-zero scalar curvature, to cite just a few papers where such examples (complete or not) can be found: $13,26,41,63,70$. 


\section{Twistor spaces With Hermitian Ricci tensor}

It is well-known that on any symplectic manifold $N$ with symplectic form $\Omega$ there exist a Riemannian metric $h$ and a $h$-orthogonal almost complex structure $J$ such that $\Omega$ is the Kähler 2 -form of the almost Hermitian manifold $(N, h, J)$, i.e., $\Omega(X, Y)=h(J X, Y)$ for $X, Y \in T N$. Recall that an almost Hermitian manifold with closed Kähler 2-form is called almost Kähler. A Riemannian metric $h$ on $N$ is said to be associated to the symplectic form $\Omega$ if there exists a $h$-orthogonal almost complex structure $J$ for which $\Omega(X, Y)=h(J X, Y)$. Note that such an almost complex structure is unique. Assume that $N$ is compact, and denote by $\mathcal{A}$ the set of all Riemannian metrics on $N$ associated to $\Omega$. If $h \in \mathcal{A}$ and $J$ is the corresponding almost complex structure, let $s$ and $s^{*}$ be the scalar curvature of the metric $h$ and the $*$-scalar curvature of the almost Hermitian structure $(h, J)$ (we recall the definition of $s^{*}$ in Section 6 ). Then we can consider the integrals

$$
\int_{N} s v_{h} \text { and } \int_{N}\left(s-s^{*}\right) v_{h} l_{h}
$$

as functionals on the set $\mathcal{A}$. D. Blair and S. Ianus 23 have proved that the critical points of both functionals are the Riemannian metrics $h \in \mathcal{A}$ whose Ricci tensor $\rho$ is Hermitian with respect to the corresponding almost complex structure $J$, i.e.,

$$
\rho(J X, J Y)=\rho(X, Y), \quad X, Y \in T N .
$$

The Kähler metrics satisfy this condition, and Blair and Ianuş raised the question of whether a compact almost Kähler manifold with Hermitian Ricci tensor is Kählerian. This question motivated the following result.

Theorem 2. (35) Let $(M, g)$ be a connected oriented real-analytic Riemannian manifold. If the Ricci tensor of the twistor space $\left(\mathcal{Z}, h_{t}\right)$ is $\mathcal{J}_{n}$-Hermitian, $n=1$ or $n=2$, then either

(i) $(M, g)$ is Einstein and self-dual

or

(ii) $(M, g)$ is self-dual with constant scalar curvature $s=12 / t$ and, for each point of $M$, at least three eigenvalues of its Ricci operator coincide.

Conversely, if $(M, g)$ is a smooth oriented Riemannian four-manifold satisfying (i) or $(i i)$, then the Ricci tensor of $\left(\mathcal{Z}, h_{t}\right)$ is $\mathcal{J}_{n}$-Hermitian.

Examples. (35]) 1. Let $M$ be an Einstein self-dual manifold with negative scalar curvature. Then, by [77, $\left(\mathcal{J}_{2}, h_{t}\right)$ for $t=-12 / s$ is an almost Kähler structure on the twistor space $\mathcal{Z}$. This structure is not Kählerian since, by the Eells-Salamon result mentioned above, the almost complex structure $\mathcal{J}_{2}$ is not integrable. On the other hand, the Ricci tensor of the metric $h_{t}$ is $\mathcal{J}_{2}$-Hermitian by Theorem 2 Thus, if $M$ is compact, the twistor space $\left(\mathcal{Z}, h_{t}, \mathcal{J}_{2}\right)$ gives a negative answer to the Blair-Ianuş question. Examples of compact Einstein self-dual manifolds with negative scalar curvature can be found in 97. Multiplying the twistor space of such a manifold by Kähler manifolds, one can construct examples of non-Kähler almost Kähler manifolds of arbitrary even dimension $\geq 6$ which have Hermitian Ricci tensors. Other examples of such manifolds can be obtained as twistor spaces of quaternionic Kähler manifolds [1]. In dimension four the Blair-Ianuş problem is not completely solved yet. In this case positive results under certain additional conditions have been proved in [10, 45, 44, 46] (see also [22, Sec. 10.2]) 
2. The Riemannian product $M=S^{1} \times S^{3}$ is a non-Einstein manifold satisfying the conditions (ii) of Theorem 2, Other examples of such manifolds can be obtained as warped-products of $S^{1}$ and $S^{3}$, see, for example, [40.

The twistor space construction can be used to obtain other examples of almost Hermitian manifolds with Hermitian Ricci tensor. Let $(M, g, J)$ be a 4-dimensional almost Hermitian manifold with the orientation induced by the almost complex structure $J$. Then $J$ is a section of the positive twistor bundle $\pi: \mathcal{Z}_{+} \rightarrow M$. Taking the horizontal lift of $J$ and the complex structure of the fibre of $\mathcal{Z}_{+}$we define an almost complex structure $\mathcal{J}$ compatible with the metrics $h_{t}, t>0$. More precisely, for $\sigma \in \mathcal{Z}, X \in T_{\pi(\sigma)} M$, and $V \in \mathcal{V}_{\sigma}$ we set

$$
\mathcal{J} X_{\sigma}^{h}=(J X)_{\sigma}^{h}, \quad \mathcal{J} V=\sigma \times V .
$$

The geometric conditions for integrability of $\mathcal{J}$ have been obtained in 42 and the Gray-Hervella classes of the almost Hermitian structure $\left(h_{t}, \mathcal{J}\right)$ have been determined in 2 .

Theorem 3. ([37]) The Ricci tensor of the almost Hermitian manifold $\left(\mathcal{Z}_{+}, h_{t}, \mathcal{J}\right)$ is Hermitian if and only if the base manifold $(M, g)$ is Einstein and anti-self-dual.

Examples. (37]) According to [2, Theorem 1], the almost Hermitian structure $\left(h_{t}, \mathcal{J}\right)$ is Kähler exactly when $(M, g, J)$ is Kähler and Ricci flat. Thus, in order to construct compact non-Kähler twistor spaces $\left(\mathcal{Z}, h_{t}, \mathcal{J}\right)$ with Hermitian Ricci tensor we need examples of compact, Einstein, anti-self-dual, non-Kähler almost Hermitian manifolds $(M, g, J)$. We consider three cases according to the sign of the scalar curvature $s$ of such a manifold.

1. Case $s>0$. In this case, by the Hitchin and Friedrich-Kurke result we have mentioned, $(M, g)$ is isometric either to the 4 -sphere $S^{4}$ with the round metric or to the complex projective space $\overline{\mathbb{C P}^{2}}$ with the opposite orientation and the FubiniStudy metric. As is well-known, none of these manifolds admits an almost complex structure for topological reasons.

2. Case $s<0$. As C. LeBrun pointed out to us, Conder and Maclachlan 29 have constructed a compact orientable Riemannian manifold $(M, g)$ of constant negative sectional curvature with Euler characteristic $\chi=16$. The signature of $M$ is zero by the well-known integral formula

$$
\tau=\frac{1}{12 \pi^{2}} \int_{M}\left(\left\|\mathcal{W}_{+}\right\|^{2}-\left\|\mathcal{W}_{-}\right\|^{2}\right) \text { vol }_{g}
$$

since both half-Weyl tensors $\mathcal{W}_{ \pm}$vanish. In particular, the intersection form of $M$ is indefinite. We also have $\tau+\chi \equiv 0 \bmod 4$. Hence, by a version of Ehresmann-Wu theorem due to O. Saeki (see, for example, 74, Theorem 8 (A)], $M$ admits an almost complex structure $I$. Then, as is well-known, $M$ admits also an almost complex structure $J$ compatible with the metric of $M$. Indeed, let $g_{0}$ be a Riemannian metric on $M$ compatible with $I$, for example take $g_{0}(X, Y)=g(X, Y)+g(I X, I Y)$. Define a symmetric positive endomorphism $G$ of $T M$ by $g_{0}(G X, Y)=g(X, Y)$. Then $J=G^{-1 / 2} I G^{1 / 2}$ is an almost complex structure compatible with the metric $g$. This almost complex structure is not integrable as the following lemma shows.

Lemma 2. (37]) Every compact anti-self-dual Hermitian surface $(M, g, J)$ of nonpositive scalar curvature is Kähler and scalar flat. 
Note also that if a Riemannian manifold $(M, g)$ admits a compatible almost complex structure $J$, it possesses many such structures inducing the same orientation as $J$. This can be seen, for example, by means of the exponential map of the twistor space $\left(\mathcal{Z}_{+}, h_{t}\right)$ of $(M, g)$ endowed with the orientation induced by $J[32,37$.

3. Case $s=0$. It is a result of Hitchin 61] that every compact, Ricci flat, antiself-dual, 4-manifold is either flat, or is a $K 3$-surface, or an Enriques surface, or the quotient of an Enriques surface by a free anti-holomorphic involution. Note also that a Kähler surface is anti-self-dual if and only if it is scalar flat. This well-known fact follows from the Kähler curvature identities which imply that the eigenvalues of the half-Weyl operator $\mathcal{W}_{+}$of a Kähler surface are $s / 6,-s / 12,-s / 12$ (see, for example, 8 ) .

Now, let $(M, g, J)$ be a compact Ricci flat Kähler surface (a Calabi-Yau surface). Let $J_{u}(p)=\exp _{J(p)}[u V(p)]$ be a 1-parameter deformation of the Kähler structure $J$, where $V$ is a non-zero compactly supported section of the pull-back bundle $J^{*} \mathcal{V} \rightarrow M$. Then every $J_{u}, u \neq 0$, is non-integrable. Otherwise, by Lemma $2, J_{u}$ would be Kähler and we would have $J_{u}=J$ everywhere since $J_{u}=J$ outside of supp $V$. Hence $V=0$ on $M$, a contradiction. Thus $\left(M, g, J_{u}\right)$ is a compact, Ricci flat, anti-self-dual, strictly almost Hermitian manifold.

Finally, let us note that the twistor spaces $\left(\mathcal{Z}_{+}, h_{t}, \mathcal{J}_{u}\right)$ of the almost Hermitian 4-manifolds $\left(M, g, J_{u}\right)$ belong to the Gray-Hervella class $\mathcal{G}_{2} 2$. Moreover, by Proposition 3 and [2, Theorem 1], it follows that $\mathcal{G}_{2}$ is the only possible GrayHervella class of the twistor spaces $\left(\mathcal{Z}_{+}, h_{t}, \mathcal{J}\right)$ with Hermitian Ricci tensor.

\section{KÄHLER CURVATURE IDENTITIES ON TWISTOR SPACES}

In order to generalize results in Kähler geometry, A. Gray [55] has introduced three classes of almost Hermitian manifolds whose curvature tensor resembles that of a Kähler manifold. On an almost Hermitian manifold $(N, h, J)$, these classes are defined by the following curvature identities:

$$
\begin{aligned}
& \mathcal{A H}_{1}: R(X, Y, Z, W)=R(X, Y, J Z, J W) \\
& \mathcal{A H}_{2}: R(X, Y, Z, W)=R(J X, J Y, Z, W)+R(J X, Y, J Z, W)+R(J X, Y, Z, J W) \\
& \mathcal{A H}_{3}: R(X, Y, Z, W)=R(J X, J Y, J Z, J W),
\end{aligned}
$$

where, as usual, $R(X, Y, Z, W)=h(R(X, Y) Z, W)$ for $X, Y, Z, W \in T N$. These identities have been used in 93 for finding irreducible components of the space of curvature tensors on an Hermitian vector space under the action of the unitary group. They have also been a useful tool for characterizing the Kähler manifolds in various classes of almost Hermitian manifolds, to quote just a few papers [6, 7, 10, 85, 94, 95. Note that in the last years there has been an intensive study of Hermitian metrics which are Kähler-like in the sense that the curvature tensor of either the Levi-Civita, Chern, Bismut-Ströminger, or more generally, a Gauduchon connection 52 has the same symmetries as the curvature tensor of a Kähler metric(see, for example, [4, 101, 102]).

The next theorem gives geometric characterizations of the oriented Riemannian four-manifolds $(M, g)$ whose twistor spaces $\left(\mathcal{Z}, h_{t}, \mathcal{J}_{n}\right), n=1,2$, belong to one of the Kähler curvature classes listed above.

Theorem 4. (39]) $(i)\left(\mathcal{Z}, h_{t}, \mathcal{J}_{n}\right) \in \mathcal{A H}_{3}$ is equivalent to $\left(\mathcal{Z}, h_{t}, \mathcal{J}_{n}\right) \in \mathcal{A H}_{2}$ and holds if and only if $(M, g)$ is Einstein and self-dual, $n=1$ or 2 . 
(ii) $\left(\mathcal{Z}, h_{t}, \mathcal{J}_{1}\right) \in \mathcal{A H}_{1}$ if and only if $(M, g)$ is Einstein and self-dual with scalar curvature $s=0$ or $s=12 / t$.

(iii) $\left(\mathcal{Z}, h_{t}, \mathcal{J}_{2}\right) \in \mathcal{A H}_{1}$ if and only if $(M, g)$ is Einstein and self-dual with $s=0$,

Remarks. ([39]) 1. By a result of S. Goldberg [53], every compact almost Kähler manifold of class $\mathcal{A H}_{1}$ is Kähler, and A. Gray [55, Theorem 5.3] has raised the question of whether the same is true under the weaker condition $\mathcal{A H}_{2}$. Now, let $(M, g)$ be an Einstein self-dual four-manifold with negative scalar curvature $s$. For $t=-12 / s$, the twistor space $\left(\mathcal{Z}, h_{t}, \mathcal{J}_{2}\right)$ is an almost Kähler manifold of class $\mathcal{A H}_{2}$ by [77] and Theorem 4 . This manifold is not Kähler, since the almost complex structure $\mathcal{J}_{2}$ is never integrable. So, we have a negative answer to Gray's question.

2. Let $(M, g)$ be a Ricci-flat self-dual four-manifold. Then $\left(\mathcal{Z}, h_{t}, \mathcal{J}_{2}\right), t>0$, is a quasi Kähler manifold [77] of class $\mathcal{A H}_{1}$ which is not Kähler. Thus, the Goldberg result cannot be extended to quasi Kähler manifolds. In the case when $M=\mathbb{R}^{4}$ the twistor space is $\mathcal{Z}=\mathbb{R}^{4} \times S^{2}$ and we recover an example of A. Gray [55].

By a result of I. Vaisman [95], every compact Hermitian surface of class $\mathcal{A H}_{1}$ is Kähler. The twistor space $\left(\mathcal{Z}, h_{t}, \mathcal{J}_{1}\right)$ is a non-Kähler Hermitian manifold of complex dimension 3 and of class $\mathcal{A H}_{1}$ by [50, 16] and Theorem 4 . If $M$ is compact, then $\mathcal{Z}$ is also compact, and we see that the Vaisman result is not true in complex dimensions greater than 2.

3. If $(M, g)$ is an Einstein self-dual four-manifold with scalar curvature $s>0$, then $\left(\mathcal{Z}, h_{t}, \mathcal{J}_{1}\right), t=12 / s$, is a Kähler manifold 50 and hence of class $\mathcal{A H}_{1}$. In fact, in this case, as we have already mentioned, either $M=S^{4}$ or $M=\mathbb{C P}^{2}$, so $\left(\mathcal{Z}, h_{t}, \mathcal{J}_{1}\right)$ is either $\mathbb{C P}^{3}$ or the complex flag manifold $F_{1,2}$ with their standard Kähler structures.

\section{6. $*$-EINSTEIN TWISTOR SPACES}

It is well known that the Ricci/Chern form of a Kähler manifold is the image $\mathcal{R}(\Omega)$ of the Kähler form $\Omega$ under the action of the curvature operator $\mathcal{R} \in \operatorname{End}\left(\Lambda^{2}\right)$. For an arbitrary almost Hermitian manifold $(N, h, J)$, the 2 -form $\mathcal{R}(\Omega)$ is neither closed nor of type $(1,1)$, but it is still closely related to the Ricci form of the canonical Hermitian connection which represents the first Chern class of $(N, J)$. The tensor $\rho^{*}$ associated to $\mathcal{R}(\Omega)$ by

$$
\rho^{*}(X, Y)=\mathcal{R}(\Omega)(X, J Y)=\operatorname{Trace}(Z \rightarrow R(J Z, X) J Y)
$$

has been introduced by S. Tachibana 91, and is known in the literature as the *Ricci tensor. This tensor then appeared in almost Hermitian geometry in different contexts. For example, it has been used by A. Gray [56] for studying nearly Kähler manifolds and by F. Tricceri and L. Vanhecke 93 for describing the irreducible components of the space of curvature tensors on a Hermitian vector space under the action of the unitary group. The $*$-Ricci tensor also plays an important role in the theory of harmonic almost complex structures, developed recently by C. Wood [100.

An almost Hermitian manifold is said to be weakly $*$-Einstein if its $*$-Ricci tensor is a multiple of the metric, i.e. if the Kähler form is an eigenvector of the curvature operator. Unlike Kähler-Einstein manifolds, the multiple (usually called the $*$-scalar curvature) need not be a constant and when this holds the manifold is called $*$-Einstein. As we have already mentioned, for Kähler manifolds the Einstein 
and weakly $*$-Einstein conditions coincide, so it is natural to ask whether there is a relation between them for more general almost Hermitian manifolds. The curvature decomposition (5) implies that in real dimension four the weakly $*$-Einstein condition holds if and only if the traceless Ricci tensor is $J$-anti-invariant and the Kähler form is an eigenvector of the self-dual Weyl operator $\mathcal{W}_{+}$. Since, for a Hermitian 4-manifold, the latter condition is equivalent to $\mathcal{W}_{+}$being degenerate (see [12]), it follows from the Riemannian Goldberg-Sachs theorem [12, 78, 84 that any Einstein Hermitian metric is weakly $*$-Einstein. For almost Kähler 4-manifolds, it is still an open question whether the Einstein condition implies the weakly $*$-Einstein one, although J. Armstrong [15] has explicitly described all weakly $*$-Einstein strictly almost Kähler Einstein 4-manifolds. This, combined with a result of K. Sekigawa [86, shows that such manifolds can never be compact, so the positive answer to the question above would imply the well-known Goldberg conjecture [53] that any compact almost Kähler Einstein 4-manifold must be Kähler. In higher dimensions, the (weakly) *-Einstein condition has not been so well studied and it seems that the main reason for that is the lack of interesting examples. Because of that in 33 the authors studied the twistor spaces of oriented Riemannian 4-manifolds as a source of 6 -dimensional examples of $*$-Einstein almost Hermitian manifolds and showed that some four-dimensional results on the $*$-Einstein condition cannot be extended to higher dimensions.

The $*$-Ricci tensor $\rho_{t, n}^{*}$ of the twistor space $\left(\mathcal{Z}, h_{t}, \mathcal{J}_{n}\right), t>0, n=1,2$, can be computed in terms of the curvature of the base manifold $(M, g)$ using the formula for the sectional curvature of $\left(\mathcal{Z}, h_{t}\right)$ in Proposition 1 and the well-known expression of the Riemannian curvature tensor by means of sectional curvatures.

Proposition 6. (33]) Let $E, F \in T_{\sigma} \mathcal{Z}$ and $X=\pi_{*} E, Y=\pi_{*} F, A=\mathcal{V} E, B=\mathcal{V} F$. Then

$$
\begin{aligned}
\rho_{t, n}^{*}(E, F) & =\left[1+(-1)^{n+1}\right] g\left(\mathcal{R}(\sigma), X \wedge K_{\sigma} Y\right)-\frac{t}{2} g\left(R\left(X \wedge K_{\sigma} Y\right) \sigma, R(\sigma) \sigma\right) \\
& +\frac{t}{4} \operatorname{Trace}\left(Z \rightarrow g\left(R(X \wedge Z) \sigma, R\left(K_{\sigma} Z \wedge K_{\sigma} Y\right) \sigma\right)\right) \\
& +(-1)^{n+1} \operatorname{Trace}\left(\mathcal{V}_{\sigma} \ni C \rightarrow g\left(R(C) X, R(\sigma \times C) K_{\sigma} Y\right)\right) \\
& +\frac{t}{2}(-1)^{n} g\left(\left(\nabla_{X} \mathcal{R}\right)(\sigma), B\right)+\frac{t}{2} g\left(\left(\nabla_{K_{\sigma} Y} \mathcal{R}\right)(\sigma), \sigma \times A\right) \\
& +\left[1+(-1)^{n+1} \operatorname{tg}(\mathcal{R}(\sigma), \sigma)\right] g(A, B) \\
& +(-1)^{n+1} \frac{t^{2}}{4} \operatorname{Trace}\left(Z \rightarrow g\left(R(\sigma \times A) K_{\sigma} Z, R(B) Z\right)\right)
\end{aligned}
$$

where $K_{\sigma}$ is the complex structure on $T_{\pi(\sigma)} M$ determined by $\sigma$.

In the case when the base manifold $(M, g)$ is Einstein and self-dual the formula for $\rho_{t, n}^{*}$ simplifies significantly:

Corollary 4. Let $(M, g)$ be an Einstein self-dual 4-manifold with scalar curvature s. Then

$$
\begin{aligned}
\rho_{t, n}^{*}(E, F) & =\frac{1}{12}\left[\left(1+(-1)^{n+1}\right) s+\frac{t}{24}\left(1+(-1)^{n}\right) s^{2}\right] g(X, Y)+ \\
& +\left[1+(-1)^{n+1} \frac{t s}{6}+(-1)^{n}\left(\frac{t s}{12}\right)^{2}\right] g(A, B) .
\end{aligned}
$$


The above formulas can be used to obtain the following geometric characterization of the $*$-Einstein twistor spaces.

Theorem 5. (33) Let $(M, g)$ be an oriented Riemannian 4-manifold with scalar curvature $s$.

(i) The twistor space $\left(\mathcal{Z}, h_{t}, \mathcal{J}_{1}\right)$ is $*$-Einstein if and only if $(M, g)$ is Einstein, self-dual and $t|s|=12$,

(ii) The twistor space $\left(\mathcal{Z}, h_{t}, \mathcal{J}_{2}\right)$ is $*$-Einstein if and only if $(M, g)$ is Einstein, self-dual and $t s=6$.

A crucial role in the proof of Theorem 5 is played by the following result essentially due to C. LeBrun and V. Apostolov (private communications, 2000) which is also of independent interest.

Lemma 3. (33) There is no self-dual manifold $(M, g)$ whose Ricci operator has constant eigenvalues $(\lambda, \mu, \mu, \mu)$ with $\lambda \neq 0$ and $\lambda \neq \mu$.

Remarks. ([33] 1. A Hermitian metric on a compact complex surface $(M, J)$ is *Einstein if and only if it is locally conformally Kähler and the traceless Ricci tensor is $J$-anti-invariant [?]. In higher dimensions however the $*$-Einstein condition does not imply any of these two properties, as can be seen by considering the twistor space $\left(\mathcal{Z}, h_{t}, \mathcal{J}_{1}\right)$ of a compact self-dual Einstein manifold $(M, g)$ with negative scalar curvature $s$ and $t=-12 / s$. By Theorem 5 the 6 -dimensional Hermitian manifold $\left(\mathcal{Z}, h_{t}, \mathcal{J}_{1}\right)$ is $*$-Einstein, but is neither locally conformally Kähler [77, nor with $\mathcal{J}_{1}$-anti-invariant traceless Ricci tensor 35 .

2. By a result of V. Apostolov [5], any compact $*$-Einstein Hermitian surface of negative *-scalar curvature is Kähler. The twistorial example above shows that the analogous statement is false in higher dimensions.

3. Recall that the twistor space $\left(\mathcal{Z}, h_{t}\right)$ is an Einstein manifold if and only the base manifold $M$ is Einstein and self-dual with positive scalar curvature $s=6 / t$ or $s=12 / t$. Thus $\left(\mathcal{Z}, h_{t}, \mathcal{J}_{1}\right), t=s / 6$, is an Einstein Hermitian manifold of real dimension 6 which is neither locally conformally Kähler 77 nor $*$-Einstein (Theorem 51). Recall also that if $M=S^{4}$ or $M=\mathbb{C P}^{2}$, then $\mathcal{Z}=\mathbb{C P}^{3}$ or $\mathcal{Z}=F_{1,2}=S U(3) / S(U(1) \times U(1) \times U(1))$, and $\left(h_{t}, \mathcal{J}_{1}\right)$ for $t=12 / s$ is the standard Kähler-Einstein structure on $\mathcal{Z}$. For $t=6 / s,\left(\mathcal{Z}, h_{t}\right)$ is a Riemannian 3-symmetric space [99] and $\mathcal{J}_{2}$ is its canonical almost complex structure. In this case $\left(\mathcal{Z}, h_{t}, \mathcal{J}_{2}\right)$ is a $*$-Einstein nearly Kähler manifold by a result of Gray [56]. Note also that for $M=S^{4}$ and $t=6 / s, h_{t}$ is the "squashed" Einstein metric on $\mathbb{C P}^{3}$ 19, Example $9.83]$.

\section{Curvature properties of the Chern connection on twistor spaces}

It is well-known [73, 52 that every almost Hermitian manifold admits a unique connection for which the almost complex structure and the metric are parallel, and the $(1,1)$-part of the torsion vanishes. It is usually called the Chern connection because, in the integrable case, it coincides with the Chern connection [28] of the tangent bundle considered as a Hermitian holomorphic bundle. This connection plays an important role in (almost) complex geometry since, by the Chern-Weil theory, the Chern classes of the manifold are directly related to its curvature. Note also the classification result of Boothby [25] who proved that the compact Hermitian 
manifolds with flat Chern connection are exactly the quotients of complex Lie groups equipped with left invariant Hermitian metrics.

Motivated by the works of S. Donaldson [43] and C. LeBrun [71, V. Apostolov and T. Dragichi [1] have proposed to study the problem of existence of almost Kähler structures of constant Hermitian scalar curvature and/or type $(1,1)$ Ricci form of its Chern connection (from now on we refer to it as the first Chern form). One of our goals in 34 was to show that the twistor space of any self-dual Einstein 4-manifold of negative scalar curvature admits such an almost Kähler structure.

Given an almost Hermitian manifold $(N, g, J)$, denote by $\nabla$ the Levi-Civita connection of $h$. Then the Chern connection $\nabla^{c}$ of $(N, g, J)$ is defined by (see, for example, [58, Theorem 6.1]):

$$
\begin{gathered}
g\left(\nabla_{X}^{c} Y, Z\right)=g\left(\nabla_{X} Y, Z\right)+\frac{1}{2} g\left(\left(\nabla_{X} J\right)(J Y), Z\right) \\
+\frac{1}{4} g\left(\left(\nabla_{Z} J\right)(J Y)-\left(\nabla_{Y} J\right)(J Z)-\left(\nabla_{J Z} J\right)(Y)+\left(\nabla_{J Y} J\right)(Z), X\right)
\end{gathered}
$$

It belongs to the distinguished 1-parameter family of Hermitian connections $\nabla^{u}, u \in$ $\mathbb{R}$, defined by P. Gauduchon [52] :

$$
\begin{gathered}
g\left(\nabla_{X}^{u} Y, Z\right)=g\left(\nabla_{X} Y, Z\right)+\frac{1}{2} g\left(\left(\nabla_{X} J\right)(J Y), Z\right) \\
+\frac{u}{4} g\left(\left(\nabla_{Z} J\right)(J Y)-\left(\nabla_{Y} J\right)(J Z)-\left(\nabla_{J Z} J\right)(Y)+\left(\nabla_{J Y} J\right)(Z), X\right)
\end{gathered}
$$

The Chern connection corresponds to $u=1$, whereas for $u=-1$ we obtain the so-called Bismut (or Ströminger) connection [89, 21].

Let $\Omega$ be the Kähler 2 -form of $(N, g, J)$ and $\delta \Omega$ the co-differential of $\Omega$ with respect to $\nabla$. Denote by $\varphi$ and $\psi$ the 2 -forms on $N$ defined by

$$
\begin{gathered}
\varphi(X, Y)=\operatorname{Trace}\left(Z \rightarrow g\left(\left(\nabla_{X} J\right)(J Z),\left(\nabla_{Y} J\right)(Z)\right)\right) \\
\psi(X, Y)=\rho^{*}(X, J Y)
\end{gathered}
$$

where $\rho^{*}$ is the $*$-Ricci tensor of $(N, g, J)$.

The formula in the next lemma appears in [52] without proof and we refer the reader to 34 for its proof.

Lemma 4. The first Chern form $\gamma^{u}$ of the Gauduchon connection $\nabla^{u}$ on an almost Hermitian manifold $(N, g, J)$ is given by

$$
8 \pi \gamma^{u}=-\varphi-4 \psi+2 u d \delta \Omega
$$

Let $(M, g)$ be an oriented Riemannian 4-manifold with twistor space $\mathcal{Z}$. Denote by $D_{n}^{c}$ the Chern connection of the almost-Hermitian manifold $\left(\mathcal{Z}, h_{t}, \mathcal{J}_{n}\right), n=1,2$, and by $\gamma_{t, n}$ its first Chern form. In the case when the base manifold $(M, g)$ is self-dual, an explicit formula for $\gamma_{t, 1}$ has been given by P. Gauduchon [51. For an arbitrary oriented Riemannian 4-manifold $(M, g)$, the first Chern forms $\gamma_{t, n}$, $n=1,2$, can be computed by means of the following formula.

Proposition 7. ([34]) The first Chern form $\gamma_{t, n}$ of the twistor space $\left(\mathcal{Z}, h_{t}, \mathcal{J}_{n}\right)$, $n=1,2$, is given by

$$
2 \pi \gamma_{t, n}(E, F)=\left[1+(-1)^{n+1}\right][g(\mathcal{R}(\sigma), X \wedge Y)+g(A, \sigma \times B)]
$$

where $E, F \in T_{\sigma} \mathcal{Z}$ and $X=\pi_{*} E, Y=\pi_{*} F, A=\mathcal{V} E, B=\mathcal{V} F$. 
Now, we consider the problem of when the curvature tensor $R_{n}^{c}$ of the Chern connection $D_{n}^{c}$ is of type $(1,1)$, i.e.

$$
R_{n}^{c}\left(J_{n} E, J_{n} F\right) G=R_{n}^{c}(E, F) G
$$

for all $E, F, G \in T \mathcal{Z}$.

Proposition 8. ([34]) (i) The curvature tensor $R_{1}^{c}$ is of type $(1,1)$ if and only if the base manifold $(M, g)$ is self-dual.

(ii) The curvature tensor $R_{2}^{c}$ is of type $(1,1)$ if and only if the base manifold $(M, g)$ is Einstein and self-dual.

The next proposition solves the problem when of the Chern connections $D_{1}^{c}$ and $D_{2}^{c}$ of a twistor space have constant holomorphic sectional curvatures.

Proposition 9. (34]) (i) The Chern connection $D_{1}^{c}$ of the almost-Hermitian manifold $\left(\mathcal{Z}, h_{t}, \mathcal{J}_{1}\right)$ has constant holomorphic sectional curvature $\kappa$ if and only if $\kappa>0$, the base manifold $(M, g)$ is of constant sectional curvature $\kappa$, and $t=1 / \kappa$.

(ii) The holomorphic sectional curvature of the Chern connection $D_{2}^{c}$ of $\left(\mathcal{Z}, h_{t}, \mathcal{J}_{2}\right)$ is never constant.

\section{Holomorphic CURVATURES OF TWISTOR SPACES}

Given an almost Hermitian manifold $(M, g, J)$ one can define various types of curvatures related to the almost Hermitian structure $(g, J)$. The most important are the holomorphic sectional curvature [69] and the holomorphic, Hermitian, and orthogonal (totally real) bisectional curvatures [54, 17, 20]. These curvatures have intensively been studied on Kähler manifolds and a lot of important results have been obtained. For example, the well-known uniformization theorem for complete Kähler manifolds of constant holomorphic sectional curvature states that any such manifold is either a complex projective space $\mathbb{C P}^{n}$ with the Fubini-Study metric, a quotient of $\mathbb{C}^{n}$ with the flat metric or a quotient of the unit ball in $\mathbb{C}^{n}$ with the hyperbolic metric [69. Moreover, by the solution of the Frankel conjecture given by Mori [76] and by Siu and Yau [88, we know that the complex projective spaces are the only compact complex manifolds admitting Kähler metrics of positive holomorphic bisectional curvature. Note also that Mok [75] has proved the so-called generalized Frankel conjecture stating that any compact simply-connected Kähler manifold with nonnegative holomorphic bisectional curvature is biholomorphic to a compact Hermitian symmetric space. We refer the reader to 68, [27, 60] for analogous results under some weaker conditions on the holomorphic bisectional curvature. The case of negative holomorphic bisectional curvature is not so rigid. For example, recently To and Yeung [92] have constructed such Kähler metrics on any Kodaira surface.

In the non-Kähler case the holomorphic curvatures mentioned above are not so well studied. Complete results have been obtained only for complex dimension 2 in which case it has been proved that every compact Hermitian surface of constant holomorphic or Hermitian sectional curvature is a complex space form [9]. In higher dimensions it is still an open question posed by Balas and Gauduchon [17, 18] whether there are compact non-Kähler Hermitian manifolds of non-zero constant holomorphic sectional curvature of the Chern connection. 
8.1. Holomorphic bisectional curvature. The holomorphic bisectional curvature $H_{t, n}$ of the twistor space $\left(\mathcal{Z}, h_{t}, \mathcal{J}_{n}\right), n=1,2$, of an oriented Riemannian 4-manifold $(M, g)$ can be computed by means of Proposition 1 For the sake of simplicity we give the respective formula only in the case when the base manifold is self-dual and Einstein.

Proposition 10. ([3]) Let $(M, g)$ be a self-dual Einstein manifold with scalar curvature $s$ and let $E, F \in T_{\sigma} \mathcal{Z}$ be arbitrary $h_{t}$-unit tangent vectors with $X=\pi_{*} E$, $Y=\pi_{*} F, V=\mathcal{V} E, W=\mathcal{V} F$. Then

$$
\begin{aligned}
H_{t, n}(E, F) & =R\left(X, K_{\sigma} X, Y, K_{\sigma} Y\right)+t\|V\|^{2}\|W\|^{2} \\
& +2 t\left(\frac{s}{24}\right)^{2}\left(\|X\|^{2}\|Y\|^{2}-g(X, Y)^{2}-g\left(K_{\sigma} X, Y\right)^{2}\right) \\
& +(-1)^{n}\left(2\left(\frac{t s}{24}\right)^{2}-\frac{t s}{12}\right)\left(\|X\|^{2}\|W\|^{2}+\|Y\|^{2}\|V\|^{2}\right) \\
& +\left(2\left(\frac{t s}{24}\right)^{2}\left(1+(-1)^{n}\right)-\frac{t s}{12}\right)\left(g\left(K_{\sigma} X, Y\right) g(\sigma \times V, W)\right. \\
& \left.+(-1)^{n} g(X, Y) g(V, W)\right),
\end{aligned}
$$

where $K_{\sigma}$ is the complex structure on $T_{\pi(\sigma)} M$ determined by $\sigma$.

We next consider two particular cases of Proposition 10 .

Corollary 5. Let $(M, g)$ be a 4-manifold of constant sectional curvature and scalar curvature s. Then

$$
\begin{aligned}
H_{t, n}(E, F) & =\frac{s}{12}\left(g(X, Y)^{2}+g\left(K_{\sigma} X, Y\right)^{2}\right)+t\|V\|^{2}\|W\|^{2} \\
& +2 t\left(\frac{s}{24}\right)^{2}\left(\|X\|^{2}\|Y\|^{2}-g(X, Y)^{2}-g\left(K_{\sigma} X, Y\right)\right) \\
& +(-1)^{n}\left(2\left(\frac{t s}{24}\right)^{2}-\frac{t s}{12}\right)\left(\|X\|^{2}\|W\|^{2}+\|Y\|^{2}\|V\|^{2}\right) \\
& +\left(2\left(\frac{t s}{24}\right)^{2}\left(1+(-1)^{n}\right)-\frac{t s}{12}\right)\left(g\left(K_{\sigma} X, Y\right) g(\sigma \times V, W)\right. \\
& \left.+(-1)^{n} g(X, Y) g(V, W)\right) .
\end{aligned}
$$

Corollary 6. Let $(M, g)$ be a self-dual Einstein manifold with sectional curvature $K$ and scalar curvature $s$, and let $E \in T_{\sigma} \mathcal{Z}$ be arbitrary $h_{t}$-unit tangent vector with $X=\pi_{*} E$ and $V=\mathcal{V} E$. The holomorphic sectional curvature of $\left(\mathcal{Z}, h_{t}, \mathcal{J}_{n}\right)$ is given by

$H_{t, n}(E)=K\left(X, K_{\sigma} X\right)\|X\|^{4}+t\|V\|^{4}+\left(2\left(\frac{s t}{24}\right)^{2}\left(3(-1)^{n}+1\right)+(-1)^{n+1} \frac{s t}{24}\right)\|X\|^{2}\|V\|^{2}$

Using Proposition 1 and Corollary 6 we obtain the following.

Theorem 6. ([36]) (i) The almost Hermitian manifold $\left(\mathcal{Z}, h_{t}, \mathcal{J}_{1}\right)$ has constant holomorphic sectional curvature $\mathcal{X}$ if and only if the base manifold $(M, g)$ has constant sectional curvature $\mathcal{X}=1 / t$.

(ii) The holomorphic sectional curvature of $\left(\mathcal{Z}, h_{t}, \mathcal{J}_{2}\right)$ is never constant. 
This together with Corollary 5 implies

Theorem 7. ([3]) The holomorphic bisectional curvature of the twistor space $\left(\mathcal{Z}, h_{t}, \mathcal{J}_{n}\right), n=1,2$, of an oriented Riemannian 4-manifold $(M, g)$ is never constant.

In the next theorem, we consider the case when the base manifold $(M, g)$ is a real space form and determine all $t>0$ for which the holomorphic bisectional curvature of its twistor space $\left(\mathcal{Z}, h_{t}, J_{n}\right)$ is strictly positive. In particular, it follows that the "squashed" metric on $\mathbb{C P}^{3}$ ([19], Example 9.83) is a non-Kähler Hermitian-Einstein metric of positive holomorphic bisectional curvature. This shows that a recent result of Kalafat and Koca [66] in dimension four can not be extended to higher dimensions.

Theorem 8. ([3]) Let $(M, g)$ be an oriented Riemannian 4-manifold of constant sectional curvature.

(i) The holomorphic bisectional curvature of $\left(\mathcal{Z}, h_{t}, \mathcal{J}_{1}\right)$ is positive if and only if $0<t s<24$.

(ii) If $(M, g)$ is a flat manifold, the holomorphic bisectional curvature of $\left(\mathcal{Z}, h_{t}, \mathcal{J}_{n}\right)$ is non-negative, $n=1,2$.

As an example illustrating Theorem 8 (ii), let us consider the twistor space $\left(\mathcal{Z}, h_{1}, \mathcal{J}_{1}\right)$ of a 4 -torus $T$ with its standard flat metric. Then $Z=T \times S^{2}, h_{1}$ is the product metric and $\mathcal{J}_{1}$ is the complex structure defined by Blanchard 24]. So, the holomorphic bisectional curvature of $\left(T \times S^{2}, h_{1}, \mathcal{J}_{1}\right)$ is non-negative. Note that $\mathcal{J}_{1}$ is not a product of complex structures on $T$ and $S^{2}$.

8.2. Orthogonal bisectional curvature. The orthogonal (totally real) bisectional curvature $B$ of an almost Hermitian manifold $(N, h, J)$ is defined in [20] by

$$
B(X, Y)=h(R(X, J X) Y, J Y)
$$

for $X, Y \in T N$ such that $X \perp\{Y, J Y\}$ and $\|X\|=\|Y\|=1$. It is well known 64 that the orthogonal bisectional curvature of a Kähler manifold of complex dimension $\geq 3$ is constant if and only if the holomorphic sectional curvature is constant. So, it is natural to ask if the same holds for other classes of almost Hermitian manifolds. The next theorem shows that this is true for the twistor spaces of self-dual Einstein 4-manifolds.

Theorem 9. (3]) Let $(M, g)$ be a self-dual Einstein 4-manifold. Then its twistor space $\left(Z, h_{t}, \mathcal{J}_{n}\right)$ has constant orthogonal bisectional curvature if and only if $n=1$ and $(M, g)$ is of constant sectional curvature $\chi=1 / t$.

Remark. Let $(M, g)$ have a constant sectional curvature. Then the orthogonal bisectional curvature $B_{t, 1}$ of the twistor space $\left(\mathcal{Z}, h_{t}, \mathcal{J}_{1}\right)$ is strictly positive if and only if $0<t s<24$.

8.3. Hermitian bisectional curvature. The Hermitian bisectional curvature $H^{c}$ of an almost Hermitian manifold $(N, h, J)$ is defined as the holomorphic bisectional curvature of its Chern connection. As we have already noted, the curvature of this connection is directly related to the Chern classes of $(N, J)$. In particular, if $\gamma$ is the first Chern form of $(N, h, J)$, then for any $X \in T N$ we have 


$$
\gamma(X, J X)=\sum_{i=1}^{n} h\left(H^{c}(X, J X) E_{i}, J E_{i}\right)
$$

where $\left(E_{1}, \ldots, E_{n}, J E_{1}, \ldots, J E_{n}\right)$ is a unitary frame.

According to Theorem [7 the holomorphic bisectional curvature of the twistor space of an oriented Riemannian 4-manifold is never constant. As for the Hermitian bisectional curvature, we have the following more general result which was pointed out to us by S. Kobayashi (private communication, April 2012).

Theorem 10. ([3]) The Hermitian bisectional curvature of a Hermitian manifold of complex dimension $\geq 2$ is never a non-zero constant.

The proof of this theorem uses formula (14) for the first Chern form $\gamma$ which implies that if the Hermitian bisectional curvature of a Hermitian manifold is a non-zero constant $c$, then the manifold is Kähler. Hence it is a complex space form and the well-known formula for its curvature [69] implies that $c=0$, a contradiction. Note also that Theorem 10 gives a partial negative answer to the question of Balas and Gauduchon [17, 18] mentioned at the beginning of this section.

Remark. ([3]) Formula (14) for the first Chern form implies that if an almost Hermitian manifold has non-zero constant Hermitian bisectional curvature, then it is an almost Kähler manifold, i.e. its Kähler 2-form is closed. Hence it is natural to ask the following questions:

- Are there compact non-Kähler and non-flat Hermitian manifolds of complex dimension $\geq 3$ with vanishing Hermitian bisectional curvature?

- Are there compact non-Kähler almost Kähler manifolds of constant Hermitian bisectional curvature?

By a result of Vezzoni [96, Theorem 4.8], if $(N, h, J)$ is an almost Kähler manifold whose holomorphic and Hermitian bisectional curvatures coincide, then it is a Kähler manifold. This result can be extended to a more general class of almost Hermitian manifolds.

Theorem 11. ([3]) Let $(N, h, J)$ be an almost Hermitian manifold such that

$$
\left(\nabla_{X} J\right)(X)=\varepsilon\left(\nabla_{J X} J\right)(J X),
$$

where $\varepsilon= \pm 1$. Then its holomorphic and Hermitian bisectional curvatures coincide if and only if $(N, h, J)$ is a Kähler manifold.

Remarks. ([3]) 1. According to the Gray-Hervella terminology [59 the almost Hermitian manifolds satisfying (15) with $\varepsilon=1$ are called $G_{1}$-spaces. This class contains the Hermitian and nearly Kähler manifolds. The identity (15) with $\varepsilon=-1$ holds for almost Kähler and quasi Kähler manifolds (recall that the quasi Kähler condition is $\left.\left(\nabla_{X} J\right)(Y)+\left(\nabla_{J X} J\right)(J Y)=0\right)$.

2. The proof of Theorem 11] shows that the above mentioned result of Vezzoni for almost Kähler manifolds holds true under the weaker condition that the holomorphic and Hermitian sectional curvatures coincide.

Finally, we describe the twistor spaces whose holomorphic and Hermitian sectional curvatures coincide. 
Theorem 12. ([3]) Let $(M, g)$ be an oriented Riemannian 4-manifold. The holomorphic and Hermitian sectional curvatures of its twistor space $\left(\mathcal{Z}, h_{t}, \mathcal{J}_{n}\right)$ coincide if and only if $(M, g)$ is a self-dual Einstein manifold with $t s=12$ for $n=1$ and

$t s=6$ for $n=2$.

\section{REFERENCES}

[1] B. Alexandrov, G. Grantcharov, and S. Ivanov, "Curvature properties of twistor spaces of quaternionic Kähler manifolds", J. Geom. 62 (1-2)), 1-12 (1998).

[2] D. Ali, J. Davidov, and O. Mushkarov, "Compatible almost complex structures on twistor spaces and their Gray-Hervella classes", J. Geom. Phys. 75, 213-229 (2014).

[3] D. Ali, J. Davidov, and O. Mushkarov, "Holomorphic curvatures of twistor spaces", Int. J. Geom. Methods Mod. Phys. 11 (3), 1450022 (2014).

[4] D. Angella, L. Ugarte, and R. Villacampa, "On Gauduchon connections with Kähler-like curvature", arXiv:1809.02632 1 [math.DG] 7 Sep 2018.

[5] V. Apostolov, "Compact *-Einstein Hermitian surfaces of negative type", C. R. Acad. Bulgare Sci. 48 (11-12), 19-22 (1995).

[6] V. Apostolov, J. Armstrong, and T. Draghici, "Local rigidity of certain classes of almost Kähler 4-manifolds", Ann. Glob. Anal. Geom. 21 (2), 151-176 (2002).

[7] V. Apostolov, J. Armstrong and T. Draghici, "Local models and integrability of certain almost Kähler 4-manifolds", Math. Ann. 323 (4), 633-666 (2002).

[8] V. Apostolov and J. Davidov, "Compact Hermitian surfaces and isotropic curvature", Ill. J. Math. 44 (2), 438-451 (2000).

[9] V. Apostolov, J. Davidov, and O. Muskarov, "Compact self-dual Hermitian surfaces", Trans. Amer. Math. Soc. 348 (8), 3051-3063 (1996).

[10] V. Apostolov and T. Draghici, "Almost Kähler 4-manifolds with $J$-invariant Ricci tensor and special Weyl tensor", Q. J. Math. 51 (3), 275-294 (2000).

[11] V. Apostolov and T. Draghici, "The curvature and the integrability of almost-Kähler manifolds: A survey", in Symplectic and Contact Topology: Interactions and Perspectives (Am. Math. Soc., Providence, RI, 2003), Fields Inst. Commun. 35, pp. 25-53.

[12] V. Apostolov and P. Gauduchon, "The Riemannian Goldberg-Sachs theorem", Int. J. Math. 8 (4), 421-439 (1997).

[13] V. Apostolovand P. Gauduchon, "Self-dual Einstein Hermitian four-manifolds", Ann. Sc. Norm. Super. Pisa, Cl.Sci, Ser. 5, 1 (1), 203-243 (2002).

[14] V. Apostolov, G. Grantcharov, and S. Ivanov, "Hermitian structures on twistor spaces", Ann. Global. Anal. Geom. 16 (3), 291-308 (1998).

[15] J. Armstrong, "An ansatz for almost Kähler, Einstein 4-manifolds", J. Reine Angw. Math. 542, 53-84 (2002).

[16] M.F. Atiyah, N.J. Hitchin, and I.M. Singer, "Self-duality in four-dimensional Riemannian geometry", Proc. Roy. Soc. Lonodon, Ser.A 362, 425-461 (1978).

[17] A. Balas, "Compact Hermitian manifolds of constant holomorphic sectional curvature", Math.Z. 189, 193-210 (1985)

[18] A. Balas and P. Gauduchon, "Any Hermitian metric of constant non-positive (Hermitian) holomorphic sectional curvature on a compact complex surface is Kähler", Math. Z. 190, 39-43 (1985).

[19] A. L. Besse, Einstein Manifolds (Springer-Verlag, 1987).

[20] R. Bishop and S. Goldberg, "Some implications of the generalized Gauss-Bonnet theorem", Trans. Am. Math. Soc. 112, 508-535 (1964).

[21] J.-M. Bismut, "A local index theorem for non-Kähler manifolds", Math. Ann. 284 (4), 681-699 (1989).

[22] D.E. Blair, Riemannian Geometry of Contact and Symplectic Manifolds (Birkhäuser, Boston, 2002), Prog. Math. 203.

[23] D.E. Blair, S. Ianuş, "Critical associated metrics on symplectic manifolds", in Nonlinear Problems in Geometry: Proc. AMS Spec. Sess., 1985, Ed. by D. DeTurck (Am. Math. Soc., Providence, RI, 1986), Contemp. Math. 51, pp. 23-29.

[24] A. Blanchard, "Sur les variétés analytique complexes", Ann. Sci. 'Ec. Norm. Supér, Sér 3, 73, 157-202 (1956). 
[25] W. M. Boothby, "Hermitian manifolds with zero curvature", Mich. Math. J. 5, 229-233 (1958).

[26] D.M.J. Calderbank and H. Pedersen, "Selfdual Einstein metrics with torus symetry", J. Diff. Geom. 60 (3), 485-521 (2002).

[27] X.X. Chen, "On Kähler manifolds with positive orthogonal curvature", Adv. Math. 215 (2), 427-445 (2007).

[28] S.-s. Chern, Complex Manifolds without Potential Theory, 2nd. ed. (Springer, New York, 1979).

[29] M. Conder and C. Maclachlan, "Compact hyperbolic 4-manifolds of small volume", Proc. Am. Math. Soc., 133 (8), 2469-2476 (2005).

[30] J.E. D' Atri and H.K. Nickerson, "Divergence-preserving geodesic symmetries". J. Diff. Geom 3, 467-176 ( 1960).

[31] J. Davidov, "Einstein condition and the twistor space of compatible partially complex structures", Diff. Geom. Appl. 22 (2), 159-179 (2005).

[32] J. Davidov, "Harmonic almost Hermitian structures", in Special Metrics and Group Actions in Geometry: Proc. INdAM workshop "New Perspectives in Differential Geometry", Rome, 2015 (Springer, Cham, 2017), Springer INdAM Ser. 23, pp. 129-159.

[33] J. Davidov, G. Grantcharov, and O. Mushkarov, "Twistorial examples of *-Einstein manifolds", Ann. Global. Anal. Geom. 20 (2), 103-115 (2001).

[34] J. Davidov, G. Grantcharov, and O. Mushkarov, "Curvature properties of the Chern connection of twistor spaces", Rocky Mt. J. Math. 39 (1), 27-47 (2009).

[35] J. Davidov and O. Mushkarov, "Twistor spaces with Hermitian Ricci tensor", Proc. Am. Math.Soc. 109, (4), 1115-1120 (1990).

[36] J. Davidov and O. Mushkarov, "On the Riemannian curvature of a twistor space", Acta Math. Hung." 58 (3-4), 319-332 (1991).

[37] J. Davidov and O. Mushkarov, "Twistorial examples of almost Hermitian manifolds with Hermitian Ricci tensor", Acta Math. Hung. 156 (1), 194-203 (2018).

[38] J. Davidov, O. Mushkarov and G. Grantcharov, "Almost complex structures on twistor spaces", in Almost Complex Structures: Proc. Int. Workshop, Sofia, 1992 (World Scientific, Singapore, 1994), pp. 113-149.

[39] J. Davidov, O. Mushkarov, and G. Grantcharov, "Kähler curvature identities for twistor spaces", Ill. J. Math. 39 (4), 627-634 (1995).

[40] A. Derdzinski, "Classification of certain compact Riemannian manifolds with harmonic curvature and non-parallel Ricci tensor", Math. Z. 172, 273-280 (1980).

[41] A. Derdziński, "Exemples de métriques de Kähler et d'Einstein autoduales sur le plan complexe", in Géométrie riemannienne en dimension 4, Sémin. Arthur Besse 1978/79, Ed. by L. Bérard-Bergery, M. Berger, and C. Houzel (CEDIC/ Fernand Nathan, Paris, 1981), pp. 334-346.

[42] G. Deschamps, "Compatible complex structures on twistor spaces", Ann. Inst. Fourier 61 (6), 2219-2248 (2011).

[43] S.K. Donaldson, "Remarks on Gauge theory, complex geometry and 4-manifolds topology", in Fields Medallists' Lectures, Ed. by M. Atiyah and D.Iagolnitzer (World Scientific, Singapore, 1997), pp. 384-403.

[44] T. C. Draghici, "On some 4-dimenasional almost Kähler manifolds", Kodai Math. J. 18 (1), 156-168 (1995).

[45] T. C. Draghici, "On the almsot Kähler manifolds with Hermitian Ricci tensor", Houston J. Math. 20 (2), 293-298 (1994).

[46] T. C. Draghici, "Almost Kähler 4-manifolds with $J$-invariant Ricci tensor", Houston J. Math. 25 (1), 133-145 (1999).

[47] J. Eells and S. Salamon, "Twistorial constructions of harmonic maps of surfaces into fourmanifolds", Ann. Sc. Norm. Super. Pisa, Cl. Sci., Ser. 4, 12, 89-640 (1985).

[48] J. Fine and D. Panov, "Symplectic Calabi-Yau manifolds, minimal surfaces and the hyperbolic geometry of the conifold", J. Diff. Geom. 82 (1), 155-225 (2009).

[49] T. Friedrich and R. Grunewald, "On Einstein metrics on the twistor spaces of a fourdimensional Riemannian manifold", Math. Nachr. 123, 55-60 (1985).

[50] T. Friedrich and H. Kurke, Compact four-dimensional self-dual Einstein manifolds with positive scalar curvature, Math. Nachr. 106, 271-299 (1982). 
[51] P. Gauduchon,"Structures de Weyl et théorèmes d'annulation sur une variété conforme autoduale", Ann. Sc. Norm. Super. Pisa Cl. Sci, Ser. 418 (4), 563-629 (1991).

[52] P. Gauduchon, "Hermitian connections and Dirac operators", Boll. Unione. Mat. Ital., Ser. 7 B 11 (2 suppl.), 257-288 (1997).

[53] S. I. Goldberg, "Integrability of almost Kähler manifolds". Proc. Am. Math. Soc. 21, 96-100 (1969).

[54] S. Goldberg and S. Kobayashi, "Holomorphic bisectional curvature", J. Diff. Geom. 1, 225-233 (1967).

[55] A. Gray, "Curvature identities for Hermitian and almost Hermitian manifolds", Tôhoku Math. J. 28, 601-612 (1976).

[56] A. Gray, "The structure of nearly Kähler manifolds", Math. Ann. 223, 233-248 (1976).

[57] A. Gray, "Einstein-like manifolds which are not Einstein", Geom. Dedicata 7, 259-280 (1978).

[58] A. Gray, M. Barros, A.M. Naveira, and L. Vanhecke, "The Chern numbers of holomorphic vector bundles and formally holomorphic connections of complex vector bundles over almost-complex manifolds", J. Reine Angew. Math. 314, 84-98 (1980).

[59] A. Gray and L. Hervella, "The sixteen classes of almost Hermitian manifolds and their linear invariants", Ann. Mat. Pura Appl., Ser. 4 123, 35-58 (1980).

[60] H. Gu and Z. Zhang, "An extension of Mok's theorem on the generalized Frankel conjecture", Sci. China Math. 53 (5), 1253-1264 (2010).

[61] N.J. Hitchin, "Compact four-dimensional Einstein manifolds", J. Diff. Geom. 9, 435-441 (1974).

[62] N.J. Hitchin, "Kählerian twistor spaces", Proc. London Math.Soc., Ser. 3, 43, 133-150 (1981).

[63] N.J. Hitchin, Twistor spaces, Einstein metrics and isomonodromic deformations, J. Diff. Geom. 42 (1), 30-112 (1995).

[64] C.-S. Houh, "On totaly real bisectional curvature", Proc. Am. Math. Soc. 56, 261-263 (1976).

[65] W. Jelonek, "Self-duality in $\mathcal{A}$-manifolds", J. London Math. Soc., Ser. 258 (3), 697-708 (1998).

[66] M. Kalafat and C. Koca, "On the curvature of Einstein-Hermitian surfaces", Ill. J. Math. 62 (1-4)(2018), 25-39 (2018).

[67] U-H. Ki and H. Nakagawa, " A characterization of the Cartan hypersurfaces in a sphere", Tôhoku Math. J., Ser.2, 39, 27-40 (1987).

[68] U-H. Ki and Y. J. Suh, "On semi-Kähler manifolds whose totally real bisectional curvature is bounded from below", J. Korean Math. Soc. 33 (4), 1009-1037 (1996).

[69] S. Kobayashi and K. Nomizu, Foundations of Differential Geometry (J. Wiley \& Sons, Hoboken, NJ, 2009), Vol. 2.

[70] C. LeBrun, "On complete quaternionic-Kähler manifolds", Duke Math. J. 63 (3), 723-743 (1991).

[71] C. LeBrun, "Ricci curvature, minimal volumes, and Seiberg-Witten theory", Invent. Math. 145 (2), 279-316 (2001).

[72] A.J. Ledger, "Harmonic Riemannian spaces", Ph.D. thesis (Univ. Durham, Durham, 1954).

[73] A. Lichnerowicz, Théorie globale des connexions et des groupes d'holonomie, (Ed. Cremonese, Roma, 1955).

[74] Y. Matsushita, "Fileds of 2-planes and two kinds of almost complex structures on compact 4-dimensional manifolds", Math. Z., 207 (2), 281-291 (1991).

[75] N. Mok, "The uniformization theorem for compact Kähler manifolds of nonnegative holomorphic bisectional curvature", J. Diff. Geom. 27 (2), 179-214 (1988).

[76] S. Mori, "Projective manifolds with ample tangent bundles", Ann. Math., Ser. 2, 110, 593-606 (1979).

[77] O. Muškarov, "Structures presque hermitiennes sur des espaces twistoriels et leurs type", C. R. Acad. Sci., Paris, Sér 1, 305, 307-309 (1987).

[78] P. Nurowski, "Einstein equations and Cauchy-Riemann geometry", PhD Thesis (SISSA/ISAS, Trieste, 1993).

[79] B. O'Neill, "The fundamental equations of a submersion", Mich. Math. J. 13, 459-469 (1966). 
[80] H. Pedersen and P. Tod, "The Ledger curvature conditions and D'Atri geometry", Diff. Geom. Appl. 11 (2), 155-162 (1999).

[81] R. Penrose, "Twistor theory, its aims and achievements", In Quantum Gravity,: An Oxford symposium (Clarendon Press, Oxford, 1975), pp. 268-407.

[82] R. Penrose, "The twistor programme", Rep. Math. Phys., 12 (1), 65-76 (1977).

[83] M. Pontecorvo, "On twistor spaces of anti-self-dual Hermitian surfaces", Trans. Am. Math. Soc. 331 (2), 653-661 (1992).

[84] M. Przanowski and B. Broda," Locally Kähler gravitational instantons", Acta Phys. Polon. B 14 (9), 637-661 (1983).

[85] T. Sato, "On some compact almost Kähler manifolds with constant holomorphic sectional curvature", in Geometry of Manifolds: Coll. Pap. 35th Symp. Diff. Geom., Matsumoto, 1988, Ed. by K. Shiohama (Academic, Boston, 1989), Prespect. Math. 8, pp. 129-139.

[86] S. Sekigawa, "On some 4-dimensional compact Einstein almost Kähler manifolds", Math. Ann. 271, 333-337 (1985).

[87] I. M. Singer and J. A. Thorpe, "The curvature of 4-dimensional Einstein spaces", in Global Analysis: Papers in Honor of K. Kodaira (Princeton Univ. Press, Princeton, NJ, 1969), Princeton Math. Ser. 29, pp. 355-365.

[88] Y.-T. Siu and S.-T. Yau,"Compact Kähler manifolds of positive bisectional curvature", Invent. Math. 59, 189-204 (1980).

[89] A. Ströminger, Superstrings with Torsion, Nucl. Phys. B 274 (2), 253-284 (1986).

[90] Z.I. Szabo, "Spectral theory for operator families on Riemannian manifolds", in Differential Geometry, Part 3: Riemannian Geometry: Proc. Summer Res. Inst., Los Angeles, 1990, Ed. by R. Green and S.-T. Yau (Am. Math. Soc., Providence, RI, 1993), Proc. Symp. Pure Math. 54, Part 3, pp. 615-665.

[91] S.-I. Tachibana "On almost-analytic vectors in almost-Kählerian manifolds", Tôhoku Math. J., Ser. 2, 11 (3), 351-363 (1959).

[92] W.-K. To and S.-K. Yeung, "Kähler metrics of negative holomorphic bisectional curvature on Kodaira surfaces", Bull. London Math. Soc. 43 (3), 507-512 (2011).

[93] F. Tricerri and L. Vanhecke, "Curvature tensors on almost Hermitian manifolds", Trans. Am. Math. Soc. 267, 365-398 (1981).

[94] I. Vaisman, "Some curvature properties of locally conformally Kähler manifolds", Trans. Am. Math. Soc. 259, 439-447 (1980).

[95] I. Vaisman, "Some curvature properties of complex surfaces", Ann. Mat. Pure Appl., Ser. 4 132, 1-18 (1982).

[96] L. Vezzoni, "On the Hermitian curvature of symplectic manifolds", Adv. Geom. 7 (2), 207-214 (2007).

[97] A. Vitter, "Self-dual Einstein manifolds", in Nonlinear Problems in Geometry: Proc. AMS Spec. Sess., 1985, Ed. by D. M. DeTutck (Am. Math. Soc., Providence, RI, 1986), Contemp. Math 51, pp. 113-120.

[98] T.J. Willmore, Riemannian geometry (Clarendon Press, Oxford, 1993.

[99] J. A. Wolf and A. Gray, "Homogeneous spaces defined by Lie group automorphisms I, II", J. Diff. Geom. 2, 77-114, 115-159 (1968).

[100] C. Wood, "Harmonic almost-complex structures", Compos. Math. 99 (2), 183-212 (1995).

[101] B. Yang and F. Zheng, "On curvature tensors of Hermitian manifolds", Commun. Anal. Geom. 26 (5), 1195-1222 (2018).

[102] S.-T. Yau, Q. Zhao, and F. Zheng, "On Ströminger Kähler like manifolds with degenerate torsion", arXiv:1908.05322 v1 [math.DG].

Johann Davidov, Institute of Mathematics and Informatics, Bulgarian Academy of Sciences, Acad. G.Bonchev Str. Bl.8, 1113 Sofia, Bulgaria

Email address: jtd@math.bas.bg

Oleg Mushkarov, Institute of Mathematics and Informatics, Bulgarian Academy of Sciences, Acad. G.Bonchev Str. Bl.8, 1113 Sofia, Bulgaria,

$$
\text { AND }
$$

Sauth-West University, 2700 Blagoevgrad, Bulgaria

Email address: muskarov@math.bas.bg 